\title{
Emotional and Physical Distress Relief Using a Novel Endorphinergic Formulation
}

\author{
Steven Crain ${ }^{1 *}$, Matthew A. Crain ${ }^{2}$, Stanley M. Crain ${ }^{3}$ \\ ${ }^{1}$ Therapeutic Alliance, State College, USA \\ ${ }^{2}$ Duke University, Durham, USA \\ ${ }^{3}$ Department of Neuroscience, Albert Einstein College of Medicine, Yeshiva University, New York, USA \\ Email: ${ }^{2}$ DrStevenCrain@aol.com
}

Received August 1, 2013; revised September 1, 2013; accepted September 14, 2013

Copyright (C) 2013 Steven Crain et al. This is an open access article distributed under the Creative Commons Attribution License, which permits unrestricted use, distribution, and reproduction in any medium, provided the original work is properly cited.

\begin{abstract}
While the endogenous opioid system has generally been associated with regulation of pain, it also modulates the experience of distress and may play a central role in many psychiatric and neurodevelopmental disorders. Decades of preclinical research on the analgesic effects of endogenous opioids, i.e., endorphins, suggests that opioid receptors have plastic bimodal (inhibitory/excitatory) properties that may explain conflicting findings in clinical research. An exploratory study with 60 healthy volunteer participants, using a cold pressor-induced pain paradigm, found evidence that a combination of a nutraceutical agent that enhances endorphin release (Endorphin Enhancer) with one that switches opioid receptors from an excitatory to inhibitory mode (Opioid Receptor Switcher) not only increases pain tolerance but also reduces emotional and physical distress. This discovery led to clinical application of a critically formulated endorphinergic treatment in 203 case studies over a two-year period. Findings revealed the remarkable clinical efficacy and safety of this treatment in the relief of chronic emotional and physical distress, including anxiety, anger, depression, cravings, and hyperalgesia, as well as enhancement of well-being, productivity, mental clarity, relationships, and an adaptive response to life's stresses. These studies provide new insights into the role of endogenous opioid system imbalances in the development, treatment, and prevention of dysfunctional emotional and physical distress. We postulate that an Endorphinergic Distress Syndrome (EDS) consists of abnormal endorphin levels together with opioid receptors predominately in their excitatory mode. EDS may account for many core distress symptoms associated with chronic anxiety, addictions, pain, as well as affective personality, autism spectrum, attention-deficit, and distress-related medical problems. Our research has led to new endorphinergic formulations, combining Endorphin Enhancers, such as caffeine, with Opioid Receptor Switchers, such as n-acetylcysteine, for the relief of emotional and physical distress. Our studies also provide a novel method to reverse the anxiogenic effects of caffeine and related hyperexcitatory substances.
\end{abstract}

Keywords: Distress; Endorphins; Anxiety; Caffeine; N-Acetylcysteine; Endorphinergic Distress Syndrome

\section{Introduction}

There is significant evidence that the endogenous opioid system is involved in attenuation of the experience of pain [1]. Endogenous opioids, i.e., endorphins, have also been implicated in the modulation of emotional and physical distress, the core component of a wide variety of psychiatric and neurodevelopmental disorders including chronic anxiety, anger, addictions, and pain as well as affective, personality, autism spectrum, and attentiondeficit disorders [2-5]. For instance, Merenlender-Wagner et al. [2] suggest that the "pathways for stress-related psychiatric disorders, depression and PTSD, converge to

"Corresponding author. a common pathway in which $\beta$-endorphin is a modulating element of distress." Hebb et al.'s [4] systematic review concludes that endogenous opioids play a central role in the modulation and regulation of the stress response and, therefore, in psychopathology. However, they note that "conflicting reports on the functional roles of endogenous opioids in stress regulation are likely to reflect the fact that different opioid systems may have opposite actions depending on the site of action, the subtype of opioid receptor involved, or the stressor conditions."

Endorphins have been found to reduce the experience of emotional and physical distress including pain $[4,6]$. In addition, there is evidence that they have rewarding 
and reinforcing properties that promote hedonia and motivation, including the popular "runner's high" $[7,8]$. Therefore, it has been hypothesized that endorphin depletion may result from prolonged stress and trauma, leading to an increase in distress, anxiety, and depression [9]. However, clinical findings have often been inconclusive and, at times, contradictory [4]. For instance, depressed patients as well as autistic children, who have marked emotional distress and impaired reward responses, often have abnormally high levels of endorphins [10,11]. While this increased endorphin release may be the nervous system's attempt to handle marked stress, this does not explain why these individuals continue to experience heightened levels of distress. Furthermore, agents that are known to increase endorphins, such as cyclic AMPphosphodiesterase (cAMP-PDE) inhibitors (e.g., caffeine) $[12,13]$, often paradoxically increase anxiety rather than relieve distress [14]. Therefore, there is a critical need to understand these contradictory findings regarding the impact of endorphins on regulation of the stress response, and specifically, on modulation of the experience of distress. This is particularly important since chronic emotional and physical distress is at the core of most psychological disorders. However, there has been little progress made toward understanding these contradictory findings or "opposite actions" of the endogenous opioid system, nor their implications for stress regulation and psychopathology.

The preclinical nerve tissue research of Crain and Shen $[15,16]$ over the past three decades on the function of opioid receptors in the pain response may provide critical insights into these issues with important clinical implications [17]. They discovered that whereas opioid receptors are normally in an inhibitory mode, thereby producing analgesia when triggered by the release of endorphins, opioid receptors could also be switched to an excitatory mode, which literally stimulates the opposite effect, that is, hyperalgesia, or increased pain sensitivity. Crain and Shen $[18,19]$ also discovered specific agents that switch opioid receptors from the excitatory (pain-sensitizing) to inhibitory (pain-reducing) mode, such as ultra-low-dose opioid antagonists (e.g., naltrexone) and sulfate-enhancing substances (e.g., sodium sulfate) [20,21]. According to Crain \& Shen [20], increased endorphin release can trigger excitatory opioid receptor signaling, thereby producing greater pain sensitivity (i.e. hypersensitivity or hyperalgesia). By combining an Endorphin Enhancer, such as a cAMP-PDE inhibitor (e.g., caffeine, rolipram), with an Opioid Receptor Switcher (e.g., ultra-low-dose naltrexone (ULDN), sodium sulfate), Crain and Shen [20,21] discovered, in their mouse tail-flick pain studies, that hyperalgesia is converted into analgesia. These studies demonstrated, for the first time, that the pain-relieving power of endorphins could be unmasked when opioid receptors are switched to the inhibitory mode. Crain and Crain [17] provide an extensive review of these preclinical pain studies on the bimodal nature of opioid receptor signaling in the endogenous opioid system and emphasize the significance of these studies in elucidating neuropharmacologic and neurochemical mechanisms that may mediate clinical distress disorders.

\section{Endorphinergic Attenuation of Physical and Emotional Distress: Cold-Pressor Studies}

\subsection{Purpose and Method}

The initial phase of this clinical investigation was designed to test the pain-relieving effects of Crain and Shen's endorphinergic cotreatment formulation, combining an Endorphin Enhancer with an Opioid Receptor Switcher, in humans, using a "cold pressor" pain assay [21]. This method is widely used to evaluate analgesic potency of pain medications [22]. For this investigation, two Jeio Tech Refrigerating Bath Circulators were used. Participants were asked to keep their non-dominant hand fully submerged in $2^{\circ} \mathrm{C}$ water, touching the bottom of the tank as long as possible. Pain tolerance, considered the most reliable behavioral measure of analgesic potency, was assessed by the duration of time the subject kept their hand submerged in the ice-cold water, with a limit set at 4 minutes to prevent potential injury. In addition, pain threshold was measured by the time to the participant's initial report of pain, while a 10-point color-graduated Visual Analogue Scale (VAS) assessed pain intensity. This procedure was conducted at baseline, and then repeated 30,60, and 90 minutes after the experimental agent (or placebo) was administered. Using a double-blind design, each subject was given a placebo, an Endorphin Enhancer alone, an Opioid Receptor Switcher alone, or the two agents together, administered in identical capsules.

For these cold pressor trials, healthy volunteer participants were recruited from the Penn State University community. Eight potential participants were eliminated from the trials since their baseline pain tolerance time was more than 2 minutes, a standard criterion used in cold pressor testing to assess meaningful experimental effects. The final 60 participants ( 38 males, 22 females; ages 18 86) went through each experimental condition, separated by at least a two-day "wash-out" period. A variety of Endorphin Enhancer and Opioid Receptor Switcher agents (and doses) were assessed in these trials, using Crain and Crain's rationale for selection of appropriate agents in each class [17,19-21]. The present report is focused on the nutraceutical agents used in these trials, all of which are considered as Generally Recognized as Safe (GRAS) by the FDA and do not require further FDA approval or a 
prescription. While our findings were generally consistent among all agents studied, this analysis highlights caffeine (100 mg), a PDE inhibitor that enhances endorphins by boosting cAMP levels (Endorphin Enhancer), combined with N-acetylcysteine (NAC) (600 mg), an amino acid that metabolizes into sodium sulfate thereby switching opioid receptors from an excitatory to inhibitory mode (Opioid Receptor Switcher) [17].

\subsection{Results}

Throughout these cold pressor trials, none of the pain measures were reduced from baseline, more than placebo, by the separate administration of the Endorphin Enhancer or the Opioid Receptor Switcher. In fact, the Endorphin Enhancers, such as caffeine, produced an average $12 \%$ decrease in pain tolerance from baseline in contrast to an average $7 \%$ increase produced by the placebo. Therefore, PDE inhibitors, such as caffeine, generally triggered hyperalgesia and an increase in pain sensitivity and reactivity. However, as predicted by our bimodal theory of endogenous opioid receptor signaling, the addition of an Opioid Receptor Switcher, such as NAC, to the Endorphin Enhancer reversed this hyperalgesia and produced a dramatic average $18 \%$ increase in pain tolerance, significantly more than the placebo. In fact, a number of the participants were able to keep their hands in the icecold water for the 4-minute protocol limit when taking certain cotreatment formulations. It is remarkable that this endorphinergic nutraceutical formulation effectively and safely increased pain tolerance, at levels similar to that seen in prior studies with acetaminophen or lowdose exogenous opiate pain medication [23,24], even though it contained no conventional analgesics. Also noteworthy is that these formulations did not significantly alter pain thresholds or pain intensity ratings. Therefore, the therapeutic benefit of this novel endorphinergic nutraceutical appears to be its ability to allow participants to tolerate or adapt to the painful stimuli, and not necessarily alter the nociceptive experience of the pain triggered by the cold water.

Moreover, clinical observations by Dr. Steven Crain (a neuropsychotherapist with a $\mathrm{PhD}$ in clinical psychology and 35 years of clinical experience) of the emotional state of the participants in these "cold pressor" studies revealed unexpected, dramatic, and meaningful changes in the distress they experienced when facing this stressinducing situation. When participants were administered a combination of an Endorphin Enhancer, such as caffeine, and an Opioid Receptor Switcher, such as NAC, a remarkable reduction in emotional and physical distress-including anxiety and agitation as well as anger and irritability - was evident in the majority of the participants, especially those who initially were the most apprehensive about the procedure. Most participants, when given this combination of agents, were clearly more relaxed and calmer with a greater sense of well-being and a more adaptive approach to the stress-induced "cold pressor" paradigm. Their cognitive functioning was intact with no side effects reported. In discussing their experience in each experimental condition, it became clear that the participants' higher pain tolerance, i.e., their ability to keep their hands in the ice-cold water longer, when administered the combination formulation was generally due to their reduced emotional and physical distress rather than a significant decrease in nociceptive pain.

\subsection{Discussion}

These clinical "cold pressor" findings cast a new light on the earlier preclinical pain studies of Crain and Shen (Section 1), suggesting that reduced behavioral reactivity to pain-induction paradigms produced by our endorphinergic nutraceutical formulation is related more to emotional and physical distress relief than analgesia. This interpretation is consistent with observations made regarding exogenous opioids as well [25]. Therefore, both endogenous and exogenous opioids appear to have a major impact on the way we handle stress, providing an ability to stay calm and less reactive and impulsive in the face of perceived threat. Clinical evidence strongly suggests, however, that reliance on endogenous opioids for the relief of emotional and physical distress is much preferred over exogenous opioids, given the serious and at times fatal side effects of exogenous opioids, including marked cognitive impairments, gastrointestinal symptoms, as well as tolerance and dependence and, ironically, hyperalgesia or increased pain sensitivity. Since the experience of dysfunctional emotional and physical distress is central to most clinical neuropsychiatric disorders, our clinical study suggests that we may have discovered a safe nutraceutical treatment for a variety of emotional and physical distress symptoms and conditions.

\section{Endorphinergic Attenuation of Emotional and Physical Distress: Clinical Case Studies}

\subsection{Purpose and Methods}

In order to explore the potential clinical implications of these exciting discoveries and insights, we initiated a 26month clinical case study investigation, assessing the benefits of this novel endorphinergic nutraceutical formulation for individuals suffering from distress-related conditions. Through direct treatment and close clinical supervision by Dr. Steven Crain, 203 case studies (121 females, 82 males, ages 14 - 85) were conducted at Therapeutic Alliance, an outpatient psychotherapy practice he established in 1980. The key criterion for participation in 
this investigation was moderate-to-severe emotional and/ or physical distress that was clearly excessive and maladaptive in relation to the individual's life circumstances. In other words, their level of distress was significantly out of proportion to the actual stress in their life. This criterion was chosen since the most dramatic benefits of the endorphinergic formulation used in the cold pressor trials were observed in participants who initially were the most distressed about the stress-induced paradigm during baseline assessment. The assumption underlying this criterion is that individuals with dysfunctional distress have unbalanced endogenous opioid systems and would benefit the most from our endorphinergic formulation. Participants suffered from a wide variety of distress-related symptoms and conditions including chronic anxiety and agitation, anger and irritability, cravings and addictions, aches and pains, as well as affective, autism spectrum, personality, and attention-deficit disorders, and distressrelated medical conditions. Most of these participants had already been in intensive psychotherapy and many had either previously tried or were concurrently on psychiatric medications including anxiolytic, anti-depressant, stimulant, and pain medication as well as a variety of herbal nutraceuticals.

The nutraceutical endorphinergic formulation used in these case studies consisted of one or more Endorphin Enhancers combined with one or more Opioid Receptor Switchers. At times, other nutraceutical agents were added to the formulation, which are thought to synergize with the endorphinergic formulation and further enhance functioning of the endogenous opioid system (Endorphinergic Synergizers). For most participants, the formulation included caffeine (60 - $280 \mathrm{mg}$ daily) and NAC (240 $1200 \mathrm{mg}$ daily). For those particularly sensitive to the stimulating effects of caffeine, the Endorphin Enhancer was changed to forskolin (60 - $240 \mathrm{mg}$ daily). At times, a second Opioid Receptor Switcher, magnesium sulfate (60 - $480 \mathrm{mg}$ daily) was added to the formulation. When needed for additional benefits, one or more Endorphinergic Synergizers were added, including L-theanine (60 $280 \mathrm{mg}$ daily) to further reduce agitation or white willow bark (200 - $800 \mathrm{mg}$ daily) to reduce pain and inflammation. The participants took our endorphinergic formulation from a minimum of two months up to the entire 26 months of the research project. At times, the endorphinergic treatment was discontinued briefly and/or modified in order to more effectively assess benefits, withdrawal response, and dosing effects. Participants were also switched, at times, to single agents in order to compare their individual effects to the endorphinergic formulation. Given the extensive history with most of these participants as well as the clear documentation of the effects of prior treatment (psychotherapeutic, pharmaceutical, nutraceutical), potential placebo effects were minimized and sepa- rated from experimental treatment effects.

\subsection{Results: Clinical Observations}

A remarkable and reliable set of clinical benefits of the endorphinergic formulation were reported by participants as well as observed by their psychotherapists, family members, friends, and co-workers. Nearly everyone reported at least some reduction in emotional and physical distress when using our endorphinergic formulation. Most participants felt an increased sense of calm, comfort, well-being, and positive mood while simultaneously experiencing enhanced mental clarity, energy, memory, and attention. For many participants these benefits began within the first day of taking the formulation, and continued with no signs of tolerance throughout the entire course of daily treatment. If anything, the benefits generally increased gradually over time. A consistent effect among most participants was a reduction in underlying agitation and restlessness, anxieties and worries, obsessions and compulsions, anger and irritability, cravings and substance abuse, as well as motivation and attention deficits. They simply felt more at peace with themselves and the world. With less fear and anger, participants were more able to use their cognitive abilities to approach life's challenges, instead of their prior tendency to respond emotionally and impulsively. A general trend was that the greater the participant's initial dysfunctional distress, the greater the benefits from the endorphinergic formulation.

As a result, most participants were more able to constructively change their maladaptive beliefs and behaviors, and often developed healthier and more active lifestyles, with greater control over unhealthy eating habits as well as drug, tobacco, and alcohol use, obsessions and compulsions, emotional outbursts, and social withdrawal. Desired weight loss was more easily obtained through healthier food choices and exercise. A dramatic reduction in relationship conflicts was evident, especially in couples and families. In fact, the most impressive reports came from other family members who appreciated the greater calm and constructive problem solving and perspective taking, and often encouraged the participant to continue using the endorphinergic formulation. In fact, during the investigation, there were many requests from family members and friends to take the nutraceutical formulation after observing the benefits in the participants and they were often added to the study. Overall, participants reported a more adaptive response to stressful life events. Many participants were able to reduce and/or eliminate their use of pharmaceutical and overthe-counter drugs, including anxiety, depression, pain, and stimulant medication. In fact, nearly everyone in the study eliminated his or her use of prescription anxiolytic medications while taking our endorphinergic formulation. 
While at least some relief of emotional and physical distress was evident in nearly all participants, many of their problems were not immediately resolved simply by taking the endorphinergic formulation. Maladaptive beliefs, values, behaviors, and mood often continued and required further treatment, including psychotherapy, coaching, skill training, and certain medications, such as antidepressant, mood stabilizing, stimulant, and anti-psychotic drugs. For instance, the endorphinergic formulation did not automatically change irrational beliefs, such as the faulty assumption that "my plane will crash." However, the reduction in emotional distress created a much more effective mind-set to allow the individual to constructively reflect on the likelihood of this catastrophic event. In another example, after an experience with his wife's betrayal, a man could continue to perceive her as the "enemy" while using the formulation, but it facilitated his ability to forgive her for her actions, since he was not as viscerally angry. Similarly, the decision to stop overeating or drinking was not evoked by the formulation, yet it made it easier to make this choice when cravings and anxiety were reduced. While physical activity, such as running, exercise, and yoga are well-known for their mood-enhancing benefits, the endorphinergic formulation facilitated participants' ability to develop these healthy lifestyles. Therefore, it became clear that these dysfunctional patterns were more easily modified when emotional and physical distress was reduced by the endorphinergic formulation. Furthermore, the more changes participants were able to make in their maladaptive beliefs, values, and behaviors as a result of their reduced emotional and physical distress while taking the endorphinergic formulation, the greater their overall long-term improvement in life satisfaction and functioning.

This comprehensive set of benefits produced by our endorphinergic formulation had not been experienced or observed with other forms of treatment by the participants or their therapists, or when any single agent in the formation was administered alone. While synergizing agents, such as L-theanine (for agitation) or white willow bark (for inflammation), enhanced certain benefits, the simple combination of an Endorphin Enhancer with an Opioid Receptor Switcher was sufficient to produce substantial emotional and physical distress relief for most participants. For certain participants who were particularly sensitive to the stimulating effects of caffeine, the Endorphin Enhancer was switched to another agent, such as forskolin, which generally provided desired benefits. At times, adding a second Endorphin Enhancer and/or Opioid Receptor Switcher, such as magnesium sulfate, improved benefits for particularly resistant symptoms of distress.

Of particular importance is that these remarkable benefits were produced with almost no reports of significant side-effects typically seen in treatments with current psychoactive pharmaceuticals and nutraceuticals, including sedation, agitation, fogginess, gastrointestinal distress, and general malaise. There was no evidence of tolerance, withdrawal symptoms, or other signs of dependence observed throughout these case studies. Moreover, it is striking that our novel endorphinergic formulations simultaneously promoted calm, comfort, and well-being, together with enhanced mental clarity, energy, and motivation. Current psychoactive pharmaceuticals and nutraceuticals generally produce a tranquilizing or energizing benefit, often with related side-effects (sedation or agitation). By contrast, our critically formulated nutraceutical preparation, by restoring normal balance to the endogenous opioid system, appears to significantly promote healthy and adaptive emotional, cognitive, and behavioral functioning, without the sedation, agitation, or fogginess, as well as tolerance and dependence typical of most current treatments.

\subsection{Results: Distress Dysfunction Inventory}

In order to quantify the benefits of our endorphinergic formulations, a Distress Dysfunction Inventory (DDI) was developed and given to each participant at the end of his or her participation in the study to complete and submit anonymously. The DDI contains a set of 22 distress dysfunction symptoms and conditions including "emotional distress," "physical distress," "worries and fears (anxiety)," "depressed mood," "aches and pains," "anger and irritability," and "obsessions and compulsions" as well as 9 functional characteristics including "calm and well-being," "physical comfort," "energy and motivation," and "concentration and attention." Participants were asked to rate the degree of symptom change, from "reduced" to "increased," as well as the change in functioning, from "improved" to "declined," on a 7-point Likert scale. All participants anonymously completed and returned the DDI. Scores were summarized to determine the percent of participants who experienced a change in symptoms and conditions as well as the percent of participants who experienced a change in functioning.

Following an extended period (range: 2 - 26 months; mean: 8 months) of generally daily use of our endorphinergic formulation, all of the 203 participants reported increased calm and well-being as well as concentration and attention, while more than $90 \%$ of them experienced an increase in emotional, relationship, sexual, work, and spiritual satisfaction. Nearly $90 \%$ of the participants reported increased physical comfort, while 75\% of them experienced increased energy and motivation. More than $90 \%$ of the participants, who suffered from emotional distress, anxiety, obsessions and compulsions, depressed mood, anger and irritability, as well as rest- 
lessness and agitation prior to commencing the endorphinergic formulation, experienced a reduction in symptoms at the end of treatment. The same level of benefits were reported by sufferers of physical distress, aches and pains, as well as concerns about motivation, energy, concentration, and attention. Similarly, more than $90 \%$ of the participants suffering from sleep, sexual, weight, and body image concerns as well as food, alcohol, and drug cravings reported at least some improvement from taking the endorphinergic formulation. Finally, nearly $90 \%$ of those suffering from memory concerns and $80 \%$ of those suffering from gastrointestinal concerns experienced at least some improvement in these problems. These selfreport DDI findings are consistent with clinical observations of these individuals and demonstrate the remarkable benefits of our nutraceutical endorphinergic formulations across a wide variety of neuropsychiatric disorders.

\subsection{Discussion}

Most participants, regardless of their neuropsychiatric diagnosis, experienced this comprehensive set of emotional and physical distress relief benefits from our nutraceutical endorphinergic formulations. These nutraceuticals appear to provide a safe method to enhance well-being, mental clarity, positive mood, and adaptive emotional, cognitive, behavioral, and physiological functioning. Obviously, simply taking these nutraceuticals did not typically resolve all the individual's problems, but they clearly helped most of them feel better and engage in their lives more adaptively as well as more effectively and thoughtfully work through their remaining issues. Given the wide variety of disorders, from chronic anxiety and addiction to autism and dementia, these findings are impressive and support the existence of an underlying imbalance in the endogenous opioid system, responsible at least in part for the participant's emotional and physical distress.

Inspired by the success of these clinical case studies at Therapeutic Alliance, case studies using our new endorphinergic formulation were conducted at several independent healthcare outpatient practices with similar benefits. More recently, endorphinergic nutraceutical formulations, based upon these discoveries, have been selfadministered by thousands worldwide. An analysis of DDI online survey responses from a sample of 307 of these individuals found a set of benefits almost as remarkable as that experienced under close clinical supervision (www.PonderaPharma.com). Therefore, these novel endorphinergic formulations appear to be therapeutic in both clinical treatment settings as well as more general populations. While these clinical case and survey studies were not blinded nor compared to placebos, the similarity of benefits in a variety of clinical settings, the close clinical observations by highly trained clinicians, as well as their consistency with the earlier double-blind "cold pressor" studies (Section 2) supports the validity of the effectiveness of this new generation of endorphinergic formulations in the treatment of emotional and physical distress across a wide variety of neuropsychiatric and neurodevelopmental disorders. These clinical findings are also consistent with the decades of preclinical nerve tissue culture and animal research on the endogenous opioid system (Section 1).

\section{Endorphinergic Distress Syndrome}

Our clinical studies provide important validation of the bimodal opioid receptor theory of the endogenous opioid system (Section 1) and the clinical impact of excessive excitatory opioid receptor signaling and abnormal and unstable endorphin availability. It is well accepted in the clinical and research literature that caffeine is counterindicated for anxiety and other psychiatric disorders since it tends to stimulate emotional distress and agitation [14, 26]. Paradoxically, by combining caffeine with an Opioid Receptor Switcher, such as NAC, as predicted by the bimodal opioid receptor theory, the anxiety stimulating and hyperalgesic effects of caffeine were transformed into anxiolytic and pain relief benefits. This reversal of caffeine's effect on emotional distress and pain sensitivity is consistent with, and supports the principle that sulfated agents, such as NAC and magnesium sulfate, can switch opioid receptors from the excitatory to the inhibitory mode (Section 1), thereby reducing anxiety and pain triggered by elevated endorphins (stimulated by cAMPPDE inhibitors, such as caffeine). Without an Opioid Receptor Switcher, administration of an Endorphin Enhancer, such as caffeine, alone is more likely to produce the opposite effects, i.e. emotional and physical distress, especially for individuals already predisposed to anxiety. We are not aware of any other current theory that can explain these clinical findings.

Therefore, this line of clinical and preclinical research supports the existence of an Endorphinergic Distress Syndrome (EDS), manifested clinically by a variety of emotional and physical distress symptoms, created and maintained, at least in part, by a dysfunctional imbalance in bimodal opioid receptor signaling and abnormal endogenous opioid levels. We postulate that EDS is a common neurophysiologic dysfunction that underlies many previously considered distinct psychiatric and neurodevelopmental disorders including chronic anxiety, addictions, pain, as well as affective, personality, autism spectrum, attention-deficit, and distress-related medical problems. EDS is a dysregulation of the stress response, which produces maladaptive fear, anger, cravings, and hyperalgesia. The high prevalence of EDS may explain the frequent resistance of many emotional and physical distress symp- 
toms, including chronic anxiety, depression, addiction, and pain to conventional treatment to conventional treatment. We are not aware of any current therapy that directly addresses EDS, or attempts to resolve these underlying imbalances in the endogenous opioid system.

It is beyond the scope of this article to systematically examine the etiological factors responsible for the development of EDS, though chronic stress, trauma, genetics, drug and alcohol use, as well as other lifestyle choices may be significant factors. In particular, chronic stress and trauma appear to trigger prolonged endorphin release as an adaptive coping response, which over an extended period of time is likely to lead to mechanisms that switch opioid receptors to a protracted excitatory mode as well as depleted and/or unstable endorphin availability. It is theorized that this shift toward opioid receptor excitatory mode is part of an adaptive expectation of danger or threat in the world, based on a prolonged experience of stress or trauma. However, even when the stress and trauma are over, this neurophysiologic adaptive coping mechanism may remain, leaving the individual prone to emotional and physical distress, i.e., EDS. Genetic factors are also very important in the etiology of EDS since many people are more "wired" toward hyperexcitability and are highly reactive to perceived stressful situations, leading to a "fight-flight" coping response, including a maladaptive tendency toward fear, anger, aggression, withdrawal, and cravings, even when circumstances do not warrant this.

There is significant evidence that widely used substances, including certain drugs, alcohol, and foods, create serious and often chronic imbalances in the endogenous opioid system, potentially leading to the development of EDS. Exogenous opioid drugs, such as oxycodone, hydrocodone, and morphine, are the most widely prescribed medications in history, and their use, both legal and illegal, continues to escalate [27]. However, their noxious and even life-threatening side effects, including tolerance, dependence, and addiction, are wellknown [28], and appear to result from their dysfunctional impact on the endogenous opioid system, including protracted excitatory receptor signaling and endorphin depletion $[16,17,29]$, thereby producing EDS [17]. In addition, the extensive use of acetaminophen may also contribute to the development of EDS since it tends to lower sulfate levels [30], which may result in excessive opioid receptor signaling, potentially involved in the wellknown hyperalgesic "rebound headache" syndrome. It is also interesting to note that NAC is the principle treatment for acetaminophen overdose and reverses this depletion of sulfates [30]. Therefore, our culture's strong reliance on pain relieving drugs, both prescription and over-the-counter, may be contributing to the development of EDS, especially among genetically vulnerable individuals. In addition, the extensive use of PDE inhibitors for respiratory conditions (e.g., theophylline), especially PDE4 inhibitors (e.g., roflumilast), which are particularly potent cAMP enhancers (and therefore Endorphin Enhancers) [20], may iatrogenically trigger EDS, including common side effects such as anxiety, depression, and insomnia.

Nicotine and other widely used substances appear to have a similar impact on the endogenous opioid system as exogenous opioids. Nicotine has been found to modify opioid receptors and alter the synthesis and release of $\beta$-endorphin and other endogenous opioids, which is responsible, at least in part, for nicotine dependence and withdrawal [31]. Alcohol consumption can also produce dependence, which has been linked to its tendency to induce endogenous opioid release [32]. Furthermore, there is growing evidence that other popular substances, including gluten in wheat and casein in milk, contain exorphins, morphine-like compounds [33,34], which have similar deleterious impact as opiates on the endogenous opioid system [17]. In addition, the widespread consumption of caffeine may be a significant etiological factor, since we provide evidence that caffeine, without co-administration of an Opioid Receptor Switcher, produces excessive excitatory opioid receptor signaling, leading to EDS symptoms such as anxiety and agitation (Sections 1-3). Excitatory amino acids, such as aspartic acid (a constituent of aspartame) and the salt of glutamic acid (MSG), widely used as additives in foods and beverages, also appear to have similar toxic impacts on the endogenous opioid system [17]. Surprisingly, excessive dietary sugar has been found to result in endogenous opioid dependence $[35,36]$, which may be due to a shift of opioid receptors to the excitatory mode, thereby producing EDS [17]. Excessive use of these extremely popular foods, beverages, and drugs appear to wreck havoc on the endogenous opioid system, producing EDS and a wide variety of chronic emotional and physical distress symptoms, including substance dependence, withdrawal symptoms, and cravings.

Another factor that may be contributing to the development of EDS is our culture's excessive avoidance of sunlight, due to cancer fears as well as long indoor work hours. According to Seneff et al. [37], reduced exposure to sunlight produces sulfate depletion, which according to our bimodal endorphinergic theory, leads to excessive excitatory opioid receptor signaling. In addition, exercise is known to naturally enhance the release of endorphins [8], yet is rarely a significant part of daily life for most people in our culture. Therefore, a number of lifestyle choices, including widely used pain relieving drugs and other medications, alcohol and tobacco, processed foods and beverages, and limited exercise and exposure to natural sunlight may all play a role in the development of 
EDS. Obviously, many people may be more vulnerable to the dysfunctional impact of these lifestyle choices due to trauma and prolonged stress as well as genetic factors.

\section{Treatment of EDS}

Our preclinical and clinical findings suggest that EDS may be responsible for many of the core distress symptoms seen in a wide variety of psychiatric and neurodevelopmental disorders. If there is indeed a common neuropathology underlying these conditions, interventions that specifically target this dysfunction may be effective across a wide variety of previously considered distinct disorders. Therefore, therapeutic methods which restore healthy balance to the endogenous opioid system by concomitantly enhancing endorphin release and switching opioid receptor signaling from an excessively excitatory to a normal inhibitory mode are expected to attenuate emotional and physical distress in these clinical disorders.

Clinical research and experience suggest that effective healthy lifestyle choices, including adaptive stress management strategies, positive outlook on life, routine exercise and exposure to sunlight, minimal use of prescription and OTC pain drugs as well as alcohol and nicotine, and a diet focused on whole natural foods and beverages (e.g., low gluten, casein, dietary sugar, sugar substitutes, and MSG) are among the best natural methods to prevent and treat EDS. However, our case studies reveal how difficult it is to maintain these healthier behaviors and attitudes, since the neurophysiologic imbalances underlying EDS create such emotional and physical distress that it is challenging to relax sufficiently to rationally handle stress adaptively. This is especially problematic for people who have experienced trauma and chronic stressful life circumstances as well as those genetically prone toward endogenous opioid system imbalances.

In addition, EDS produces a dependency on, and cravings for substances that dysfunctionally impact upon the endogenous opioid system, including alcohol, nicotine, pain drugs, processed sugar, unbalanced caffeinated beverages, "comfort foods," and certain food additives. In fact, when opioid receptors are switched to a protracted excitatory mode, methods that stimulate endorphins, such as exercise, are more likely to produce distress than pleasure, leading to avoidance of healthy activity. This vicious cycle potentiates EDS since unbalanced endogenous opioid systems lead us to experience emotional and physical distress. This increased distress greatly enhances our interpersonal conflicts, maladaptive stress-coping strategies, and cravings for comfort foods, beverages, and drugs, thereby exacerbating our anxiety, anger, cravings, depression, hyperalgesia, aggression, and withdrawal. Our case studies reveal that, at times, this vicious cycle can only be broken by methods that directly restore healthy ho- meostatic balance in the endogenous opioid system. Therefore, our endorphinergic formulation appears to be necessary to treat EDS for many people who suffer from emotional and physical distress.

Ever since the discovery of opioid receptors and "endogenous morphine," or "endorphins," as Eric Simon named them, many have envisioned that the "Holy Grail" would be therapeutic regulation of these amazing "molecules of emotion" [38,39]. With our discovery of EDS and a method to restore healthy balance in the endogenous opioid system, our research offers promise of a remarkably safe and effective treatment for the emotional and physical distress symptoms underlying a wide variety of psychiatric and neurodevelopmental disorders, including chronic anxiety, anger, addictions, hyperalgesia, as well as affective, autism spectrum, attention-deficit, and personality disorders. Our endorphinergic treatment may also help to prevent and treat stress-related illnesses. By combining Endorphin Enhancers, such as caffeine and/or forskolin, to insure sufficient and consistent endorphin levels, with Opioid Receptor Switchers, such as NAC and/or magnesium sulfate, to restore normal inhibitory opioid receptor signaling, critically formulated nutraceutical and pharmaceutical preparations may provide a novel method to correct neurophysiologic imbalances in the endogenous opioid system.

It is interesting to note that our endorphinergic formulation worked so effectively with such a wide variety of distress symptoms and disorders, especially since prior studies have shown that distress conditions may involve abnormally low or high endorphin levels (Section 1). Our case studies suggest that regardless of the endorphin imbalance, the inclusion of an Endorphin Enhancer is generally beneficial. This finding may be explained by the homeostatic nature of the endogenous opioid system. Preclinical studies $[16,18]$ have shown that excitatory opioid receptor signaling tends to promote endorphin levels by increasing cyclic AMP and neurotransmitter release, which may enhance endorphin release in some parts of the nervous system. This evidence suggests that when an Opioid Receptor Switcher restores normal inhibitory signaling, endorphin production is likely to decrease. Therefore, regardless of whether endorphins initially are abnormally high or low, administration of an Endorphin Enhancer is generally needed to insure sufficient and reliable endorphin availability at the same time that normal inhibitory opioid receptor signaling is restored by the Opioid Receptor Switcher.

With a balanced endogenous opioid system, we are generally less emotionally and physically distressed and more able to respond to stressful situations appropriately and adaptively without self-defeating struggles and conflicts or reliance on "comfort" drugs and alcohol or foods and beverages. By reducing these maladaptive coping 
strategies and substances, EDS is further reduced, leading to a healthier, more satisfying and enjoyable life. However, it is important to note that while these endorphinergic nutraceuticals appear to be effective for EDS, they are not a treatment for medical disease conditions. Therefore, our endorphinergic formulations should only be considered as part of a comprehensive treatment program that targets dysfunctional emotional and physical distress. Obviously, these new generation nutraceuticals appear to have remarkable benefits for individuals who suffer from EDS, that is, an underlying imbalance in their endogenous opioid system, manifested by excessive emotional and physical distress. For these individuals, the restoration of healthy homeostatic balance in the endogenous opioid system, essentially tuning down fight-flight distress alerts, facilitates more effective "top-down" regulation between our emotion centers, where endorphins are concentrated, such as the limbic system and the gut's "second brain," and our higher cognitive processes in the prefrontal cortex. By enhancing "top-down" regulation, our endorphinergic formulation provides a therapeutic tool in the treatment of a wide variety of distress-related conditions.

It is not expected that this formulation will have much immediate benefit to those who already have a balanced endogenous opioid system, though regular use of these endorphinergic nutraceuticals may help prevent the development of EDS. Another way to prevent EDS on a large-scale basis might be the systematic addition of an Opioid Receptor Switcher, such as NAC or ULDN, in all drugs, foods, and beverages that may trigger excessive excitatory opioid receptor signaling, including opiate drugs, acetaminophen, certain respiratory medications, (e.g., cAMP-PDE inhibitors, such as roflumilast and theophylline), nicotine and alcohol products, caffeinated and sweetened foods and beverages, as well as specific food additives (e.g., excitatory amino acids, as in aspartame and MSG).

\section{Autism Spectrum Disorder: Early-Onset EDS}

Autism Spectrum Disorder (ASD) provides an excellent illustration of the application of our bimodal endorphinergic theory to a specific clinical condition since there are a number of lines of evidence that suggest that many ASD symptoms stem from early-onset EDS produced by both genetic and environmental factors. Panksepp [40] theorized that autism involves an endogenous opioid system imbalance, citing studies that autistic children often have excessively high levels of endorphins. It has been speculated that the withdrawn state of autistic children appears similar to opiate intoxication, and the agitated state is reminiscent of opiate withdrawal [11]. However, the mechanism of these changing states has not been under- stood. Studies have found that children with ASD have abnormally low sulfate levels $[41,42]$ as well as excessive GM1 ganglioside in their cerebrospinal fluid [43]. Our bimodal endorphinergic theory helps to explain these findings since excessive GM1 ganglioside together with sulfate deficiency are expected to produce protracted opioid receptor excitatory signaling [17]. Seneff et al. [37] have proposed that sulfate deficiency in ASD may be due, in part, to avoidance of sunlight exposure by pregnant mothers and infants. We also speculate that early use of acetaminophen in infants and young children, typically administered concurrently with immunizations and for medical conditions as an alternative to aspirin, may be a factor, especially for genetically vulnerable children, since this drug is known to produce sulfate deficiencies [44]. Furthermore, research suggests that ASD children have digestive system dysfunctions, which may result in excessive exorphins due to incomplete metabolism of gluten and casein [37]. Together, these findings are consistent with serious imbalances in the endogenous opioid system, including excessive excitatory opioid receptor signaling and highly variable availability of endogenous and exogenous opioids, which may explain the intense swings in emotional and physical distress symptoms in these children. These factors and the extensive consumption of certain foods such as sugar, wheat, and milk products, combined with genetic vulnerabilities [e.g., 43] including gut dysfunction, may be the "perfect storm," which may produce severe EDS and many of the distress symptoms manifested by ASD children and adults.

These biochemical assays in ASD children provide support for our bimodal endorphinergic theory suggesting that EDS may be a critical factor in producing the distress symptoms in ASD. In addition, opiate antagonists at doses that block both excitatory and inhibitory opioid receptor signaling have been shown to provide some, albeit inconsistent, benefits for ASD children, demonstrating involvement of the endogenous opioid system [45]. Furthermore, prior clinical research has found evidence of benefits of using NAC [46] for ASD, though without consistent findings or a clear explanation for the mechanism. Similarly, an elimination diet is often used in ASD, focused on gluten and casein, as well as caffeine and sugar, but again with variable benefits [47]. Our model of EDS offers an explanation as to how these interventions provide benefits, but also why they are unreliable. We postulate that effective treatment needs to include both an Opioid Receptor Switcher, such as NAC, as well as an Endorphin Enhancer, such as caffeine, to insure sufficient and reliable levels of endorphins, and to maintain inhibitory opioid receptor signaling. This treatment should reduce emotional and physical distress, as well as facilitate withdrawal from exorphins as trigger 
foods are eliminated. In fact, we found that our nutraceutical endorphinergic formulations have shown significant distress relief benefits in case studies with both children and adults with ASD, including marked reduction in agitation, obsessive-compulsive symptoms, distractibility, and withdrawal (Section 3). The use of our endorphinergic formulation also greatly facilitated the ability of these children and adults to effectively eliminate gluten, casein, and sugar from their diet without typical withdrawal symptoms as well as develop a healthy exercise routine. Our preliminary clinical findings provide support for the role of EDS in ASD as well as the use of our novel endorphinergic formulations in ASD treatment. Further research is needed to explore these EDS biochemical and neurophysiologic factors in ASD as well as later-onset EDS disorders.

\section{Endogenous Opioid System and Adaptive Stress Response}

While the endogenous opioid system plays a central role in our adaptive response to stress and imbalances in this system appear to be responsible for many distress symptoms, it has complex homeostatic inter-connections to other stress-related neurotransmitter systems, such as dopamine, glutamate, serotonin, GABA, and norepinephrine [48]. Therefore, balancing the endogenous opioid system, using endorphinergic therapeutics, can also have homeostatic benefits on the other stress-related systems. In addition, pharmaceutical and nutraceutical therapies that target other stress-related neurotransmitter systems can have positive effects on the endogenous opioid system. In fact, many conventional treatments, such as Selective Serotonin Reuptake Inhibitors (SSRI), were often helpful in conjunction with our endorphinergic formulation for participants in our clinical study. However, the evidence from our clinical case studies suggests that many symptoms of emotional and physical distress require the restoration of endogenous opioid system balance for long-term relief. Without direct intervention in the endogenous opioid system, many distress symptoms are often resistant to most conventional forms of treatment.

While our choice of NAC as an Opioid Receptor Switcher for our formulations was based on its sulfation effects on the endogenous opioid system [17,21], it also directly modulates other stress-related neurotransmitters including dopamine and glutamate [49]. Therefore, the remarkable benefits of our cotreatment formulation may actually be mediated through several important stressrelated neurotransmitter systems in addition to the endogenous opioid system. Furthermore, NAC has been shown to have therapeutic benefits for a wide variety of distress-related conditions, including obsessive-compul- sive disorder, trichotillomania, pathological gambling, bipolar disorder, schizophrenia, ASD, Alzheimer's, as well as methamphetamine, cannabis, nicotine, and cocaine dependence [50]. Although these clinical reports fail to acknowledge possible effects of NAC on the endogenous opioid system, they provide support for the use of NAC in our endorphinergic nutraceuticals, and also for our cotreatment formulations since they were remarkably more effective than NAC alone in our clinical studies. The therapeutic benefits of NAC alone, as well as our endorphinergic formulations, for such a diverse range of psychiatric and neurodevelopmental disorders strongly suggests a central pathophysiology in the stress processing center of the nervous system, which stems from neurophysiologic imbalances in the endogenous opioid and other stress-related neurotransmitter systems.

We postulate that the endogenous opioid system serves as the central alert system, informing the individual of the relative safety (inhibitory opioid receptor mode), or threat (excitatory opioid receptor mode) from the environment or one's own body. The release of endogenous opioids triggers inhibitory (calming) or excitatory (distressing) opioid receptor signaling, which then homeostatically stimulates the other neurotransmitter systems, together producing the full range of emotions, cognitions, and behaviors relevant to the specific circumstances. Dysfunctional endorphinergic imbalances, such as opioid receptors abnormally switched to the excitatory mode, reflect a dispositional bias to the perception of danger or threat, thereby producing distress symptoms of EDS. Endorphinergic therapeutics that restore healthy balance to the endogenous opioid system are likely to directly reduce these EDS symptoms. In view of the complex interconnected stress adaptation system, however, interventions that target other neurotransmitter systems, such as SSRIs, are likely to indirectly impact the endogenous opioid system, thereby producing distress-relieving benefits as well. Our theory suggests that methods which restore healthy homeostatic balance in the endogenous opioid system, including both sufficient endorphin availability and normal inhibitory opioid receptor signaling, may provide direct resolution of the central neurophysiologic dysfunction and, therefore, more extensive relief from emotional and physical distress. Once normal balance is restored in the endogenous opioid system, homeostasis is likely to promote healthy balance in the other neurotransmitter systems as well. In this way, healthy emotional, cognitive, behavioral, and physiological regulation is restored. Thus, a well-functioning balanced endogenous opioid system provides resilience and an adaptive stress response including effective higher-order cognitive functioning and a generally positive hedonic tone with less interference by more primitive "fight-flight" reactions, such as maladaptive fear, anger, and cravings. Sim- 
ply put, stress does not have to produce distress.

\section{Future Research Implications}

Obviously, these clinical studies have been exploratory and need further investigation. Future research can elucidate and validate the existence and nature of endogenous opioid system imbalances and their role in causing EDS, and the extent to which a common endorphinergic dysfunction underlies many of the distress symptoms typically associated with psychiatric, neurodevelopmental, and distress-related medical conditions. These studies could clarify which of the endogenous opioids, such as $\beta$ endorphins, and their primary locations in the nervous system, such as in specific regions in the brain or gut, are more critically involved in EDS. This research should also explore the complex relationships among the important stress-related neurotransmitter systems, including endorphin, glutamate, dopamine, GABA, norepinephrine, and serotonin in order to determine the most effective levels of intervention. The existence and nature of genetic dispositions toward EDS need to be studied, such as ASD and other conditions with early onset emotional and physical distress, and their interface with trauma, chronic life stresses, and lifestyle choices.

Systematic large-scale clinical trials as well as more targeted double-blind, placebo-controlled studies will help validate and refine the use of our novel critically formulated endorphinergic formulations, consisting of one or more Endorphin Enhancers and one or more Opioid Receptor Switchers, with possible Endorphinergic Synergizers, for the prevention and treatment of EDS. Investigation of functionally relevant nutraceutical and pharmaceutical agents and their combinations, as well as dosing, will be important. For instance, the combination of a specific cAMP-PDE4 inhibitor, such as roflumilast, with ULDN, should be considered as a potent pharmaceutical treatment for EDS. Further research is also suggested that can evaluate the benefit of these endorphinergic therapeutics as a safe method to enhance well-being, mental clarity, positive mood, and adaptive emotional, cognitive, behavioral, and physiological functioning. Finally, the longterm effects of these endorphinergic formulations need to be explored, including their lasting benefits after discontinuation. Further research is needed to study the role of changes in beliefs, behaviors, and values made while taking the formulation in facilitating and synergizing long-term benefits.

Clinical research, including neuroimaging, biochemical, and pharmacologic analyses, should be designed to systematically investigate the specific imbalances in the endogenous opioid system, including maladaptive opioid receptor signaling and endorphin levels that underlie each EDS cluster to determine whether specific neurophysiologic profiles exist. For instance, certain disorders may involve excessive endorphins, such as autism, while depleted endorphins may be implicated in other conditions, such as post-traumatic stress disorder. Similarly, while most distress conditions are likely to involve excessive excitatory opioid receptor signaling, there may be others that could stem from excessive inhibitory signaling, such as certain catatonic states. Once these specific profiles are determined, and assessment methods are established, more critically targeted endorphinergic formulations can be developed. Finally, neuroimaging, biochemical, and pharmacologic analyses can be used to determine the specific impact these endorphinergic treatments have on the central and peripheral nervous systems, especially regarding changes in "top-down" processing of stressful events.

Systematic methods need to be developed for the prevention of EDS. Studies should address the positive impact on the endogenous opioid system of teaching effective stress management techniques, especially in early childhood education. Identification of vulnerable children and adults, due to more serious stress, trauma, and genetic factors, for such programs would be important. Research is also suggested to investigate health benefits of natural endorphin enhancing lifestyle choices, such as routine exercise, relaxation, and sun exposure, as well as potential risks of drugs, foods, and beverages that may trigger excessive excitatory opioid receptor signaling, including opiate drugs, acetaminophen, certain respiratory medications, nicotine and alcohol products, caffeinated and sweetened foods and beverages, as well as specific food additives. While minimizing use of these popular substances could reduce endorphinergic imbalances, studies should also address the possible benefits of routinely adding Opioid Receptor Switchers to them in order to help prevent the development of EDS.

\section{REFERENCES}

[1] L. F. Tseng, "Mechanisms of $\beta$-Endorphin-Induced Antinociception," In: L. F. Tseng, Ed., The Pharmacology of Opioid Peptides, Harwood Academia Publishers, Newark, United States, 1995, pp. 249-269.

[2] A. Merenlender-Wagner, Y. Dikshtein and G. Yadid, "The $\beta$-Endorphin Role in Stress-Related Psychiatric Disorders," Current Drug Targets, Vol. 10, No. 11, 2009, pp. 1096-1108.

http://dx.doi.org/10.2174/138945009789735147

[3] S. C. Ribeiro, S. E. Kennedy, Y. R. Smith, C. S. Stohler and J.-K. Zubieta, "Physical and Emotional Stress Regulation through the Endogenous Opioid System and $\mu$ Opioid Receptors," Progress in Neuro-Psychopharmacology \& Biological Psychiatry, Vol. 29, No. 8, 2005, pp. 1264-1280.

http://dx.doi.org/10.1016/j.pnpbp.2005.08.011

[4] A. L. O. Hebb, S. Laforest and G. Drolet, "Chapter 4.9 Endogenous Opioids, Stress, and Psychopathology," Te- 
chniques in the Behavioral and Neural Sciences, Vol. 15, 2005, pp. 561-583.

doi.org/10.1016/S0921-0709(05)80031-8

[5] A. R. Prossin, T. M. Love, R. A. Koeppa, J. K. Zubieta and K. R. Silk, "Dysregulation of Regional Endogenous Opioid Function in Borderline Personality Disorder," American Journal of Psychiatry, Vol. 167, No. 8, 2010, pp. 925-933.

http://dx.doi.org/10.1176/appi.ajp.2010.09091348

[6] D. Parikh, A. Hamid, T. C Friedman, K. Nguyen, A. Tseng, P. Marquez and K. Lufty, "Stress-Induced Analgesia and Endogenous Opioid Peptides: The Importance of Stress Duration," European Journal of Pharmacology, Vol. 650, No. 2-3, 2011, pp. 563-567. http://dx.doi.org/10.1016/j.ejphar.2010.10.050

[7] J. Panksepp, B. H. Herman, T. Vilberg, P. Bishop and F. G. DeEskinazi, "Endogenous Opioids and Social Behavior," Neuroscience \& Biobehavioral Reviews, Vol. 4, No. 4, 1980, pp. 473-487. http://dx.doi.org/10.1016/0149-7634(80)90036-6

[8] P. C. Dinas, Y. Koutedakis and A. D. Flouris, "Effects of Exercise and Physical Activity during Depression," Irish Journal of Medical Science, Vol. 180, No. 2, 2011, pp. 319-325. http://dx.doi.org/10.1007/s11845-010-0633-9

[9] L. Hoffman, P. B. Watson, G. Wilson and J. Montgomery, "Low Plasma $\beta$-Endorphin in Post-Traumatic Stress Disorder," Australian and New Zealand Journal of Psychiatry, Vol. 23, No, 2, 1989, pp. 269-273. http://dx.doi.org/10.3109/00048678909062145

[10] G. M. Goodwin, M. P. Austin, S. M. Curran, M. Ross, N. Prentice, K. P. Ebmeier, J. Bennie, S. Carroll and H. Dick, "The Elevation of Plasma Beta-Endorphin Levels in Major Depression," Journal of Affective Disorders, Vol. 29, No. 4, 1993, pp. 281-289. http://dx.doi.org/10.1016/0165-0327(93)90018-F

[11] M. Leboyer, M. P. Bouvard, C. Recasens, M. H. Plumet, D. Walter-Perotte, F. Tabuteau, F. Bondoux and M. Dugas, "Opiate Hypothesis in Infantile Autism? Therapeutic Trials with Naltrexone," L'Encephale, Vol. 19, No. 2, 1993, pp. 95-102.

[12] M. A. Arnold, D. B. Carr, D. M. Togasaki, M. C. Pian, and J. B. Martin, "Caffeine Stimulates Release in Blood But Not in Cerebrospinal Fluid," Life Sciences, Vol. 31, No. 10, 1982, pp. 1017-1024.

[13] R. H. Khalil and M. R. Soliman, "Diazapam Alters Caffeine-Induced Effects on Beta-Endorphin Levels in Specific Rat Brain Regions," Life Sciences, Vol. 61, No. 25, 1997, pp. 2485-2490. http://dx.doi.org/10.1016/S0024-3205(97)00983-1

[14] D. M. Veleber and D. I. Templer, "Effects of Caffeine on Anxiety and Depression," Journal of Abnormal Psychology, Vol. 93, No. 1, 1984, pp. 120-122. http://dx.doi.org/10.1037/0021-843X.93.1.120

[15] K.-F. Shen and S. M. Crain, "Dual Opioid Modulation of the Action Potential Duration of Mouse Dorsal Root Ganglion Neurons in Culture," Brain Research, Vol. 491, No. 1, 1989, pp. 227-242. doi:org/10.1016/0006-8993(89)90059-0

[16] S. M. Crain and K.-F. Shen, "Modulation of Opioid An- algesia, Tolerance, and Dependence by Gs-Coupled, GM1 Ganglioside-Regulated Opioid Receptor functions," Trends in Pharmacologic Science, Vol. 19, No. 9, 1998, pp. 358365. http://dx.doi.org/10.1016/S0165-6147(98)01241-3

[17] S. Crain and S. M. Crain, "Endorphinergic Attenuation of Distress by Concomitantly Enhancing Endogenous Opioid Release and Switching Opioid Receptor Signaling from an Excessively Excitatory to a Normal Inhibitory Mode," Journal of Behavioral and Brain Research, Vol. 3 , in Press.

[18] S. M. Crain and K.-F. Shen, "Antagonists of Excitatory Opioid Receptor Functions Enhance Morphine's Analgesic Potency and Attenuate Opioid Tolerance/Dependence Liability," Pain, Vol. 84, No. 2-3, 2000, pp. 121-131. http://dx.doi.org/10.1016/S0304-3959(99)00223-7

[19] S. M. Crain and K.-F. Shen, "Neuraminidase Inhibitor, Oseltamivir Blocks GM1 Ganglioside-Regulated Excitatory Opioid Receptor-Mediated Hyperalgesia, Enhances Opioid Analgesia and Attenuates Tolerance in Mice," Brain Research, Vol. 995, No. 2, 2004, pp. 260-266. http://dx.doi.org/10.1016/j.brainres.2003.09.068

[20] S. M. Crain and K.-F. Shen, "Low Doses of Cyclic AMPPhosphodiesterase Inhibitors Rapidly Evoke Opioid-Mediated Thermal Hyperalgesia in Naïve Mice Which Is Converted to Prominent Analgesia by Cotreatment with Ultra-Low-Dose Naltrexone," Brain Research, Vol. 1231, 2008, pp. 16-24.

http://dx.doi.org/10.1016/j.brainres.2008.07.015

[21] S. Crain, W. Crain, S. M. Crain and M. Crain, "Methods and Compositions for Treating Distress Dysfunction and Enhancing Safety and Efficacy of Specific Medications," USPTO Patent 8372414, February 12, 2013.

[22] S. F. LaVincente, J. M. White, A. A. Somogyi, F. Bochner and C. B. Chapleo, "Enhanced Buprenorphine Analgesia with the Addition of Ultra-Low-Dose Naloxone in Healthy Subjects," Clinical Pharmacology \& Therapeutics, Vol. 83, No. 1, 2007, pp. 144-152.

[23] C. S. Yuan, T. Karrison, J. A. Wu, T. K. Lowell, J. P. Lynch and J. F. Foss, "Dose-Related Effects of Oral Acetaminophen on Cold-Induced Pain: A Double-Blind, Randomized Placebo-Controlled Trial," Clinical Pharmacology and Therapeutics, Vol. 63, No. 3, 1998, pp. 379-383. http://dx.doi.org/10.1016/S0009-9236(98)90169-2

[24] Z. D. Cooper, M. A. Sullivan, S. K. Vosburgm, J. M. Manubay, M. Haney, R. W. Foltin, S. M. Evans, W. J. Kowalczyk, P. A. Saccone and S. D. Corner, "Effects of Repeated Oxycodone Administration on Its Analgesic and Subjective Effects in Normal, Healthy Volunteers," Behavioral Pharmacology, Vol. 23, No. 3, 2012, pp. 271279. http://dx.doi.org/10.1097/FBP.0b013e3283536d6f

[25] G. J. McKenna, "Methadone and Opiate Drugs: Psychotropic Effect and Self-Medication," Annals of the New York Academy of Sciences, Vol. 398, 1982, pp. 44-55. http://dx.doi.org/10.1111/j.1749-6632.1982.tb39472.x

[26] C. France and B. Ditto, "Cardiovascular Responses to the Combination of Caffeine and Mental Arithmetic, Cold Pressor, and Static Exercise Stressors," Psychophysiology, Vol. 29, No. 3, 1992, pp. 272-282. http://dx.doi.org/10.1111/j.1469-8986.1992.tb01698.x 
[27] B. Meier, "Pain Killer: A Wonder Drug's Trail of Addiction and Death," Rodale Press, Emmaus, 2003.

[28] E. Nestler, "Historical Review: Molecular and Cellular Mechanisms of Opiate and Cocaine Addiction," Trends in Pharmacological Sciences, Vol. 25, No. 4, 2004, pp. 210-218. http://dx.doi.org/10.1016/j.tips.2004.02.005

[29] S. M. Crain and K.-F. Shen, "Chronic Morphine-Treated Sensory Ganglion Neurons Remain Supersensitive to the Excitatory Effects of Naloxone for Months after Return to Normal Culture Medium: An in Vitro Model of 'Protracted Opioid Dependence,"” Brain Research, Vol. 694, No. 1-2, 1995, pp. 103-110.

[30] J. T. Slattery, J. M. Wilson, T. F. Kalhorn and S. D. Nelson, "Dose-Dependent Pharmacokinetics of Acetaminophen: Evidence of Glutathione Depletion in Humans," Clinical Pharmacology and Therapeutics, Vol. 41, 1987, pp. 413-418. http://dx.doi.org/10.1038/clpt.1987.50

[31] M. Hadijconstantinou and N. H. Neff, "Nicotine and Endogenous Opioids: Neurochemical and Pharmacological Evidence," Neuropharmacology, Vol. 60, No. 7-8, 2011, pp. 1209-1220.

http://dx.doi.org/10.1016/j.neuropharm.2010.11.010

[32] J. M. Mitchell, J. P. O’Neil, M. Janabi, S. M. Marks, W. J. Jagust and H. L. Fields, "Alcohol Consumption Induces Endogenous Opioid Release in the Human Orbitofrontal Cortex and Nucleus Accumbens," Science Translational Medicine, Vol. 4, No. 116, 2012, p. 116ra6. http://dx.doi.org/10.1126/scitranslmed.3002902

[33] H. Teschemacher, G. Koch and V. Brantl, "Milk ProteinDerived Opioid Receptor Ligands," Biopolymers (Peptide Science), Vol. 43, No. 2, 1997, pp. 99-117. http://dx.doi.org/10.1002/(SICI)1097-0282(1997)43:2<99 ::AID-BIP3>3.0.CO;2-V

[34] F. R. Huebner, K. W. Lieberman, R. P. Rubino and J. S. Wall, "Demonstration of High Opioid-Like Activity in Isolated Peptides from Wheat Gluten Hydrolysates," Peptides, Vol. 5, No. 6, 1984, pp. 1139-1147.

http://dx.doi.org/10.1016/0196-9781(84)90180-3

[35] C. Colantuani, P. Rada, J. McCarthy, C. Patten, N. Avena, A. Chadeayne and B. Hoebel, "Evidence That Intermittent, Excessive Sugar Intake Causes Endogenous Opioid Dependence," Obesity Research, Vol. 10, No. 6, 2002, pp. 478-488. http://dx.doi.org/10.1038/oby.2002.66

[36] N. M. Avena, P. Rada and B. G. Hoebel, "Evidence for Sugar Addiction: Behavioral and Neurochemical Effects of Intermittent, Excessive Sugar Intake," Neuroscience and Biobehavioral Reviews, Vol. 32, No. 1, 2008, pp. 2039. http://dx.doi.org/10.1016/j.neubiorev.2007.04.019

[37] S. Seneff, R. Davidson and L. Mascitelli, "Might Cholesterol Sulfate Deficiency Contribute to the Development of Autistic Spectrum Disorder?" Medical Hypotheses, Vol. 78, No. 2, 2012, pp. 213-217. http://dx.doi.org/10.1016/j.mehy.2011.10.026

[38] C. B. Pert, "Molecules of Emotion," Scribner, New York,
1997.

[39] C. F. Levinthal, "Messengers of Paradise: Opiates and the Brain," Doubleday, New York, 1988.

[40] J. Panksepp, "A Neurochemical Theory of Autism," Trends in Neuroscience, Vol. 2, 1979, pp. 174-177.

[41] F. G. Bowling, H. S. Heussler, A. McWhinney and P. A. Dawson, "Plasma and Urinary Sulfate Determination in a Cohort with Autism," Biochemical Genetics, Vol. 51, No, 1-2, 2013, pp. 147-153.

http://dx.doi.org/10.1007/s10528-012-9550-0

[42] R. H. Waring and L. V. Klovrza, "Sulphur Metabolism in Autism," Journal of Nutritional and Environmental Medicine, Vol. 10, No. 1, 2000, pp. 25-32. http://dx.doi.org/10.1080/13590840050000861

[43] A. Lekman, O. Skjeidal, E. Sponheim and L. Svennerholm, "Gangliosides in Children with Autism," Acta Paediatrica, Vol. 84, No. 7, 1995, pp. 787-790. http://dx.doi.org/10.1111/j.1651-2227.1995.tb13757.x

[44] P. Good, "Did Acetaminophen Provoke the Autism Epidemic?” Alternative Medicine Review, Vol. 14, No. 4, 2009, pp. 364-372.

[45] G. Christopher, "Endogenous Opioids and Opiate Antagonists in Autism: Brief Review of Empirical Findings and Implications for Clinicians," Developmental Medicine \& Child Neurology, Vol. 37, No. 3, 1995, pp. 239-245. http://dx.doi.org/10.1111/j.1469-8749.1995.tb11998.x

[46] A. Y. Hardan, L. K. Fung, R. A. Libove, T. V. Obukhanych, S. Nair, L. A. Herzenberg, T. W. Frazier and R. Tirouvanziam, "A Randomized Controlled Pilot Trial of oral N-Acetylcysteine in Children with Autism," Biological Psychiatry, Vol. 71, No. 11, 2012, pp. 956-961. http://dx.doi.org/10.1016/j.biopsych.2012.01.014

[47] C. M. Pennesi and L. C. Klein, "Effectiveness of the GlutenFree, Casein-Free Diet for Children Diagnosed with Autism Spectrum Disorder: Based on Parental Report," Nutritional Neuroscience, Vol. 15, No. 2, 2012, pp. 85-91. http://dx.doi.org/10.1179/1476830512Y.0000000003

[48] G. Dichter, C. A. Damiano and J. S. Allen, "Reward Circuitry Dysfunction in Psychiatric and Developmental Disorders and Genetic Syndromes: Animal Models and Clinical Findings," Journal of Neurodevelopmental Disorders, Vol. 4, No. 19, 2012, pp. 1-43.

http://dx.doi.org/10.1186/1866-1955-4-19

[49] O. Dean, F. Giorlando and M. Berk, "N-Acetylcysteine in Psychiatry: Current Therapeutic Evidence and Potential Mechanisms of Action," Journal of Psychiatry and Neuroscience, Vol. 36, No. 2, 2011, pp. 78-86.

[50] M. Berk, G. S. Malhi, L. J. Gray and O. M. Dean, "The promise of N-Acetylcysteine in Neuropsychiatry," Trends in Pharmacological Science, Vol. 34, No. 3, 2013, pp. 167-177. http://dx.doi.org/10.1016/j.tips.2013.01.001 